\title{
Panel: Will IS development methods be completely incorporated in CASE tools in the future?
}

\section{Motivation}

Will methods disappear? The manuals of the methods can be completely integrated in CASE tools. What will the impact be on the quality of systems development? Is it really desirable to have the art of IS design reduced to mouse clicks? What does this mean for training? Shouldn't we work on a Eurotool instead of a Euromethod?

\section{Panellists}

Dr. M. Breu, Siemens-Nixdorf, München, Germany

Dr. S. Brinkkemper, University of Twente, Netherlands (Chair)

Mr. M. Franckson, Sema Group, Paris, France

Dr. H. Habrias, Université de Nantes, France

Dr. T. Halpin, University of Queensland, Australia

Prof. P. Loucopoulos, UMIST, Manchester, UK

The panellists are known for their academic and industrial contributions to the field of methods and tools for IS development. This panel aims at an attractive discussion initiated by highly interactive panellists with outspoken, possibly controversial, opinions. Conference attendees expect a state-of-the-art reflection combined with a vision on the future in IS development, from both the academic as the industrial perspective.

\section{State of the art in IS development methods}

Momentarily, IS development methods are still being revised, extended, specialised. There are national standards, company standards, and commercial methods. Well-known methods include: Information Engineering, ISAC, JSD, Merise, Method/1, Navigator, NIAM, SA/SD, SDM, and SSADM. Special development methods have been developed for expert systems, real-time and embedded systems, and standard software selection. Recently, object orientation came into the centre of interest resulting into various object oriented development methods, such as OMT and OOA/OOD. Harmonisation efforts, sponsored by organizations as the EC (Euromethod), IEEE or DoD, try to restrict the divergence of terminology and deliverable structure among the various IS development methods.

\section{State of the art in CASE tools}

Since their conception in the beginning of the eighties CASE tools have gained an established position in the market of development support tools. Well-known tools include: ADW, Excelerator, Foundation, IEF, OracleCASE, Pacbase, SDW, Software 
through Pictures, System Architect, and Teamwork. The first tools had their own graphical user interface, whereas nowadays the interface is usually conforming the platform standards of DOS/Windows, OS2/Presentation Manager, and UNIX/XWindows. The tools support the clerical documentation tasks of the developer and provide all kinds of analysis checks on the correctness of the specifications. More recently, customisable tools came on the market. Tools, such as VSF, Toolbuilder and Maestro, allow to adapt the graphical editors to the needs of the development organization. For all CASE tools aspects as performance, integration with the software engineering platforms, quantifiable retum of investment, and development process support are still problematic.

\section{Current issues}

As development projects are ranging over various dimensions, e.g. type of application, scale of systems, development approach, and organisational impact, there is a broad spectrum of current issues in methods and tools. We mention here only a few.

- To what extent can the steps and guidelines of an IS development method be incorporated in a CASE tool? What degree of methodical guidance is desirable for the average developer? The extent to which a method is supported by a tool is called the method companionship and it may be clear that this exists in various degrees.

- How can methods and tools be maintained? Will special application domains, as knowledge based systems and real-time systems, require special methods and tools? Will new IS development insights, like object orientation, require new versions and variants of the methods in the future?

- The development of a coherent terminology and guidelines for the structure and contents of milestone deliverables. How should multi-method companies or consortia merge their individual IS development practices? Harmonisation of methods need not go hand in hand with harmonisation of tool usage.

- The diversity of projects requires contingent methods, i.e. methods configured to the situation at hand. What means should be provided to have optimal CASE support for contingent methods?

\section{The future of methods and CASE}

To predict the coming years of methods and tools is hard. The various stakeholders in the market: customers, vendors, and national and international bodies, can't direct the process individually. The open market will decide on the success of new products new development approaches and new initiatives. However, the main complaint that IS development still goes out of budget and time, needs serious attention. Development should be quality engineering supported by quality tools. 